\title{
DATA FUSION APPLIED ON AUTONOMOUS GROUND VEHICLES
}

\author{
Emanuel TAROPA, \\ Computer Science Dept., \\ Yonsei University \\ etaropa $@$ yonsei.ac.kr
}

\author{
Vason P. SRINI \\ Computer Science Dept., \\ Yonsei University \\ srinivp@yonsei.ac.kr
}

\author{
Won-Jong LEE \\ Computer Science Dept., \\ Yonsei University \\ airtight $@$ yonsei.ac.kr
}

\author{
Tack-Don HAN \\ Computer Science Dept., \\ Yonsei University \\ hantack@kurene.yonsei.ac.kr
}

\begin{abstract}
Accurate environment sensing has become crucial in various domains ranging from remote control, remote collaboration, navigation in autonomous vehicles and disaster prevention systems. The common characteristics for these applications are: diverse sensors operating at different rates resulting in the heterogeneity of data sources, constant processing time constraints, and real-time decision making processes based on the collected information. A reliable data fusion model must address each of these requirements and must allow the user to constantly monitor the flow of data in the framework. This paper proposes a hierarchical model for data fusion using real time objects and an application program interface (API).
\end{abstract}

Keywords - Data fusion, autonomous navigation, real-time systems, software-engineering.

\section{Introduction}

Bob Lutz, vice chairman of GM, in his article in the fortune magazine [1] predicts that autonomous driving cars could arrive in less than two decades. But before that can happen, several important problems have to be solved. Some of them are: lane following, lane departure warning, perceptive passing, adaptive cruise control, environment sensing to determine the vehicles around and communication between cars. Some of the common tasks for the above problems are: acquiring data from sensors, processing it and applying the desired actions using actuators. For correctly executing these tasks, a permanent monitoring of the environment is necessary For interactivity and adaptability, strict timing is required in collecting, processing and interpreting the environmental data.

In the present paper we describe a hierarchical data fusion scheme able to support an Autonomous Navigation System, (ANS) by clearly separating vehicle control, environment sensing, and navigation functions. Our data fusion framework possesses flexible synchronization primitives that support a wide variety of interaction patterns among programmer defined data fusion objects. The rest of the paper is organized as follows: Section 2 presents the motivation behind hierarchical data fusion, illustrating problems posed by implementing an ANS. The functional specification is presented in Section 3. Section 4 contains the real-time API necessary for the data fusion framework and its applications for ANS. Section 5 concludes the paper, highlighting important aspects subject to future developments as well as conclusions reached during the project.

\section{Existent Work and Motivation}

The recent success of Stanford's Stanley's SUV in the DARPA [2] Grand Challenge Off-Road Robot Race proves that a good software design on commercially available vehicles far outstrips custom made solutions with inter-operability issues between the software layer and vehicle's hardware.

Autonomous driving is essentially a continuous environment sensing application producing continuous sensor data that needs to be integrated by the navigation module. This continuous stream data is generated in a regular manner, with sensor-dependent frequency and format. Although many conceptual and practical models have been researched for data fusion [3], [4] they are usually tailored for specific hardware architectures and applications. A framework specifying a general context and set of rules for writing data fusion applications has been described in [5]. Even if it supports diverse interaction scenarios among fusion nodes and has high deployment flexibility, it doesn't treat effectively the synchronization among fusion objects.

Our model of hierarchical data fusion with strict synchronization among fusion objects allows data fusion applications to be written easier and have a more compact code. The programmer can now focus on the logic of the application instead of worrying about synchronizing the clocks of the fusion system and of managing access to common resources.

\section{Functional Specification}

Breaking complex problems into smaller, easily manageable parts is one of the fundamental concepts software engineering relies on. In order to construct an easy to understand and usable data fusion framework, central points in data fusion on a collection of multiple, heterogeneous sensors need to be identified and treated separately.

The functional specification we provide for data fusion will be exemplified using an Autonomous Ground Vehicle (AGV), with an architecture described by Figure 2 . Thus we prove that even without being specifically tailored for AGV data fusion architecture, our data fusion framework is able to support complex applications in an efficient way. 


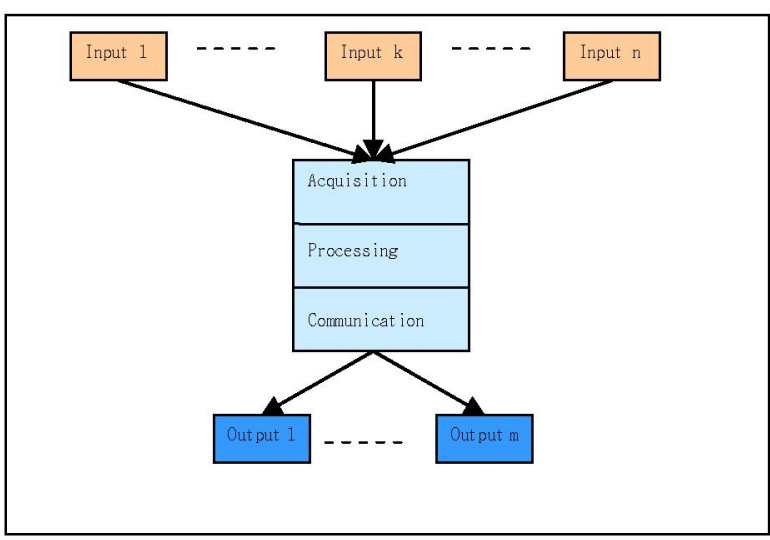

Figure 1. Decomposing Data Fusion at Fusion Node Level

When speaking about functionality inside a data fusion network, we have used the term "fusion object". Let us now link fusion objects to the hardware platforms where they are executed. We call the resulting ensemble as fusion node and we define it as follows:

\section{Definition 1 (Fusion Node)}

We term as fusion node any component of a fusion framework capable of executing data acquisition and / or data processing. Distinct fusion objects residing on the same hardware platform and performing distinct fusion functions are regarded as distinct fusion nodes.

For simplicity in specifying the flow of data within the data fusion system and for clearly identifying classic interaction patterns (e.g. client - server interaction) we connect the fusion nodes in a tree pattern, which we will call from now on fusion tree.

\section{Definition 2 (Fusion Tree)}

We understand by a fusion tree an abstract modeling of a fusion network where connections among processing acquisition nodes are represented by links and processing acquisition nodes are represented by fusion nodes.

Although graph models have been extensively used by previous work [4] we believe that a tree based modeling reveals more efficiently hierarchical relations among fusion nodes. Analyzing data fusion at a fusion node's level we notice it has the following components:

1. Acquisition - information from one or more sources is gathered

2. Processing - filters and normalization are applied on acquired data

3. Communication - processed data is sent to parent node

\subsection{Acquisition Layer}

The Acquisition Layer represents a fusion node's interface to its data provider nodes, connecting the node with an inferior level in the fusion tree. It is at this level that data conversion, data rate adjustment and data re-packetization take place. These transformations on the received data are crucial for

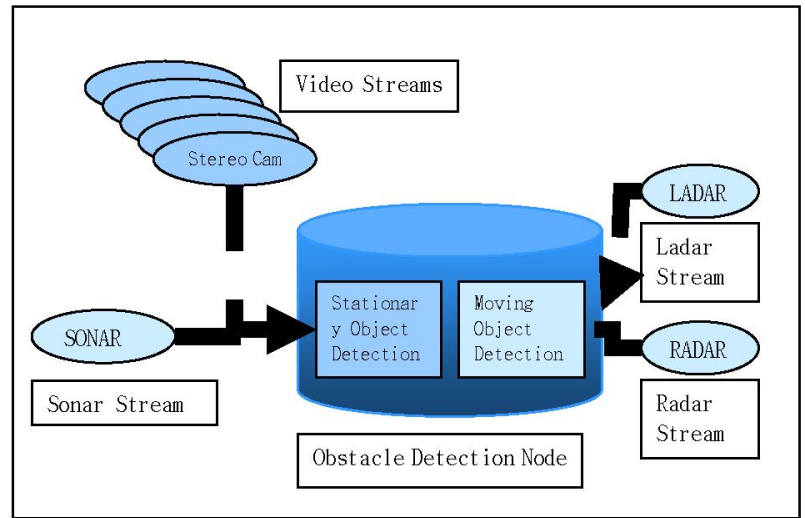

Figure 2. Autonomous Ground Vehicle Data Fusion Network

These transformations on received data are essential for effective data processing, analysis and fusion.

Considering that a processing node has more then one child in the fusion tree, the Acquisition Layer will have to manage different data streams coming from the node's children.

Without actually performing the data fusion, the acquisition layer still has to convert incoming data in the format imposed by the processing layer. As the frequencies of the incoming streams can be different, the acquisition layer has to locally buffer / compress some of the results arrived from inferior level nodes and deliver accordingly to processing's node desired rates.

For example, let's consider the simple case of the sonar device connected to the central navigation module - Obstacle Detection Node, as represented in Figure 2. As sonar represents a sensing node endowed with limited processing power, and is the primary entry point of data in the fusion, it will be designated as a leaf in the fusion tree. According to the type of sonar equipment, basic data compression and packetization can occur on the sonar node right before sending the data to its clients. In the worst case scenario, no post-measurement processing is applied on data on the sonar node before sending it to the Obstacle Detection Node. Therefore it is up to the Obstacle Detection Node to transform the sonar data in the most convenient format for processing and analysis. This transformation will happen at the Acquisition Layer level.

If now we consider the totality of data sources arriving at the Obstacle Detection Module, we notice that the streams represent different sensors: sonar, radar, ladar and video. As different sensors usually mean different sampling frequencies (i.e. video images are sampled at 30 frames / second while sonar and ladar can be configured to give readings every $5 \mathrm{~ms}$ ) and different data formats (e.g. depth extraction from video images is encoded differently than distance to the still objects resulted from ladar measurements). The acquisition layer of a node needs therefore to deal with the heterogeneity of formats and frequencies for the incoming data streams. A node must be able to understand the data formats that are presented to it and must combine correctly the information having different sampling frequencies. In section 4 we will analyze some of the functions on which the Acquisition Layer relies in its operation. 


\subsection{Processing Layer}

The processing layer comprises all the transformations that are applied on the data received from the acquisition layer. At this level the algorithms can operate either on combined data (i.e. - the stationary object detection algorithm from the obstacle detection node of our AGV operates on data containing sonar readings for the AGV's proximity and combined video images for perspective) or on simple sensor data (i.e. image normalization algorithms take as input directly the raw image).

Environment sensing applications need to have consistency between their state and the exterior state so they perform a permanent scan of the environment. This permanent scan results in continuous sensor readings that are directed toward the processing nodes of the framework. Depending on the nature of the application and the sensors used, the frequency of the sensor data can be constant or variable. Our data fusion framework architecture accepts both constant and variable frequency streams by having appropriate structures and functions for handling streams during their lifetime.

As data arrives in a continuous manner, it needs to be processed in a continuous manner. Therefore the transformations expressed by the node's processing level will be applied repeatedly on the incoming data. The result of the processing layer will be a new data stream, having a frequency depending on the execution time of the applied transformations. Considering streams with constant frequency for inputs to the node, the output needs to form also a stream with constant frequency. Therefore, for correctly processing constant frequency streams, the fusion framework must ensure that intermediate operations applied on these streams have a bounded execution time.

An efficient pipelining of operations on data streams requires that each operation in the sequence be executed at a specific time in order not to miss any data from the stream and not to be desynchronized with the other operations. Therefore, for supporting efficient pipelining of operations, a data fusion framework needs to offer a deterministic scheduling of processing layer's methods. In Section 4 we will explore the functions that the Processing Layer relies on during its operation.

\subsection{Forwarding Layer}

After constant time transformations have taken place in the processing layer over data incoming from Acquisition Layer, the results need to be forwarded to client nodes. As we previously pointed out, some incoming streams can have constant frequency and hence the resulting streams will also have constant frequency. The nodes expecting these streams assume that they have a constant frequency and therefore, schedule their operation accordingly. For perfect timing, both on the sender and the receiver sides, the communication delay between them needs to have a bounded if not constant value. Based on this value, precise estimates on the arrival of data can be made on the receiver and hence data processing methods can be deterministically scheduled.

Fully characterizing the requirements of each of the three degrees of separation presented by Figure 1 we discovered crucial parameters for any data fusion framework: the need to support heterogeneous data formats and frequencies addressed by the acquisition layer, to handle hard real time constraints with constant kernel execution time and deterministic scheduling - addressed by the processing layer and to have bounded communication time along any path in the fusion tree - addressed by the communication layer.

\section{Data Fusion API}

The hierarchical data fusion API needs to address the requirements identified in the functional description given in the above section. The main objectives followed during the API's design are:

1. Lightweight - the model needs to run on low level platforms.

2. Distributed - the processing is done on a collection of processing nodes. The only requirement is that these systems run a RTOS [6], [7] or RT-enable middleware [8]

3. Portable - the data fusion framework's processing nodes are heterogeneous (e.g. PDA's, laptops, sensor nodes). The data fusion model needs to be insensitive to OS and architecture diversity among nodes

4. Extensible - modular construction ensures ease of programming

5. Scalable - the design needs to scale well when the processing network increases in size

Data fusion API's specification resulted from analyzing both the operations at fusion objects level as well as the interactions between fusion objects.

\subsection{Real Time Object}

Real time objects (RTO) are best represented in a 3 dimensional space: data, operation and time [8]. They are classical OOP objects that have added the notion of time. AN RTO is described by adding to the basic principles of object oriented programming - encapsulation, polymorphism and inheritance we add the constraint of determined execution time for object's methods.

There are two ways of integrating the time dimension in an object oriented programming language: (1) to keep the language unchanged and to delegate an inferior layer (either the operating system or a middleware) to provide the ability of expressing real-time actions and conditions and (2) to specify a new real time object oriented programming language [9]. Regardless of choosing either (1) or (2) we assume we have RTO functionality and we write the data fusion API accordingly.

\subsubsection{Interactions between RTOs}

Objects can be classified accordingly to the interactions that exist between them in: service providers and service clients [10]. An object offering a specific functionality will be called a service provider. An object using a service will be called a client of the object that offers that service.

The most common interaction between different objects is represented by the method call. In its simplest form, a method 


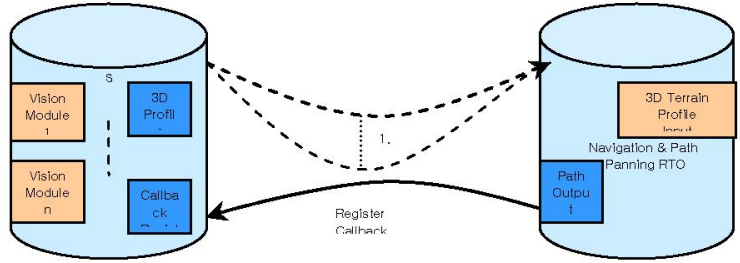

Figure 3. Subscription Interaction

of an object simply calls a method of another object. We will all from now on the first object the caller and the latter object the callee. The callee will perform the requested function and will return the result either in the locations made available by the caller or by side effect. Once the procedure call finishes, the caller will be able to use the procedure's results.

Based on the flow of actions on the caller / callee, two basic types of interactions exist: synchronous and asynchronous. For the synchronous calling scenario, the caller must block and wait until the results from the callee are available and then continue its normal processing. Asynchronous procedure invocation scenario is the following: the caller makes the procedure call to the callee and then registers a callback function. Then both caller and callee continue their processing and when the result is computed, the callee will call synchronously the callback procedure of the caller, pushing the result to it.

Dealing with continuous, time varying, data sources requires constant interaction between the caller and the callee. Therefore, two communication paradigms have been developed: polling, as illustrated by Figure 3 and subscription as represented in Figure 4.

In the polling scenario, the caller is periodically checking the client to see if there is data available for processing. This paradigm has evolved from the synchronous procedure calling. This type of interaction is currently used in email clients and other internet applications. The advantage of polling is that the load is predictable on the client. The main disadvantage is the overhead in network traffic, due to the queries for data sent by the caller.

The subscription paradigm has emerged from the asynchronous procedure calling. According to this interaction scenario, the callee is periodically pushing data to the caller (by calling the caller's callback function). The main advantage results from minimizing the network transfers between the caller and the callee. The disadvantage resides in the fact the load on the client does not remain predictable - i.e. data can arrive any time and then a context switch might be required for processing / fusing.

For example, in modeling the interaction between the higher level navigation module and the sonar data analysis module, we have two options: (1) polling - the higher level navigation module will periodically check the sonar for close by obstacles. The frequency will be high enough so that unexpected situations can be handled, even if the vehicle is at full speed and (2) subscription - the sonar will periodically send data to the higher navigation module. If an unexpected event occurs, then the callback function for the higher navigation module will be immediately invoked. Each one of these perspectives has advantages and disadvantages and the choice is ultimately application dependent.



Figure 4. Polling Inter action

\subsubsection{Guaranteed Completion Time}

An important constraint in data fusion applications is the constant time required for each of the intermediate stages of the data processing / fusing. Combining data from multiple sources on the same endpoint requires that the combined data be situated in the same temporal vicinity. Thus we can achieve an accurate description of the environment at a given moment of time.

For example, when performing depth information extraction from video images taken with a set of two cameras, we must make sure that the frames used are taken at roughly the same moment of time and that we do not try to merge information from a newer frame with information from an older one.

Real time object methods used in data fusion need to advertise to the RTOS / middleware their maximum completion time. This time is set by the programmer, taking into account the system processing capability and maximum load. More advanced schemes of load prediction / balancing can be imagined, for example dynamically changing a method's guaranteed execution time depending on the current load of the advertising peer, but such schemes are complex and need high processing power.

\subsubsection{Determined Scheduling}

The processing part of data fusion can be viewed as an aggregation of operations executed at precise time intervals. For allowing a continuous flow of information in the data fusion network, real time objects need to have their functionalities well pipelined over the same stream of data that they are processing. The guaranteed completion time constraint imposed on RTO methods is combined with the triggering time to allow maximum programming flexibility. Thus, it is possible to specify not only how long a given method will take but also when the respective method should start executing.

\subsubsection{RTO - non RTO interactions}

So far we have discussed only interactions between RTOs. The assumption that we made is that every node included in the DF framework will be running some RTOS or RT-enable middleware and have a collection of RTOs exposing a unified interface. There are some applications where one might be interested in designing a network combining both RTO and non-RTO systems, especially for supporting nodes with low processing power at the edges of the data fusion framework (e.g. 8051 microcontrollers). Therefore, an extensible architecture for a fusion network must deal with interfacing with non-RT objects. Thus, the DF logic can be kept at the core of the fusion network and data acquisition and primary 
filtering operations can take place on low power, inexpensive components.

\subsection{Synchronization Functions}

The data fusion framework (DFF) needs to ensure synchronization between RTOs located on different peers. Time can therefore be either local (local time - LT) - on each peer or global (global time - GT) - at system's level. Establishing the GT is done using a time master (TM). Synchronization signals are considered to be part of the DFF's command signals. Synchronization relies on the following functions:

\subsubsection{Setting the Global Time Master}

This function lets the programmer specify a Global Time Master (GTM). The GTM will periodically initiate DFF synchronization by sending synchronization messages (SM) to the other peers connected in the DF network (DFN). The SMs can use either a dedicated connection between the peers (CAN bus) or they can share the same connection with data messages (DM). The implementation of the communication protocol is application / architecture dependent.

Setting the GTM is done using the following parameters: target peer id, initial max drift allowed between any two RTOs, initial sync period controlling the frequency of synchronization.

Setting the GTM can be done from application level. Each RTO has a function for accepting a GTM request from the framework.

The protocol for setting the GTM is:

1. The request is transmitted to the desired IP

2. The target peer verifies the existence of a GTM in the system.

3. If a GTM has been detected at previous step, an error code is returned, specifying that there has been already specified a GTM.

4. If no GTM has been detected, then the peer broadcasts his position as a GTM to the other peers.

The peer being asked to become a GTM can take other factors in the decision process. These factors could be: the expected overhead of being GMT can not be tolerated without degrading the DF performances of the respective peer, the connection to the TM / DFN does not allow for frequent SM broadcasts to other peers.

\subsubsection{Sending Synchronization Messages}

The GTM must maintain at all times the system within the specified maximum drift (MD). Therefore, it will broadcast the time periodically to the other peers. This function resides on every RTO but is active only on the GTM. The transmission media for SMs is dependent on the application media. The modeling of continuous execution will be dependent on the RTOS / middleware used by the DF application.

The synchronization message needs to be small enough in order to allow a high frequency of broadcasts. We recommend that the synchronization message be represented only by header information._A protocol for sending synchronization messages is:
1. Send synchronization message with initial frequency

2. Estimate the MD of the system

If $\mathrm{MD}<$ maximum admissible drift (MAD) than increase the synchronization frequency and go to 1 .

Observations: Only the GTM can invoke this function

\subsubsection{Applying Synchronization Messages}

Each RTO needs to understand the SMs received from the GTM. Upon receiving a SM the RTO will synchronize its clock to the broadcasted value of time.

The synchronization could (should) take place in the future. This means that the GTM should broadcast a future time for synchronization, based on the network delays and synchronization speed on each peer. The network paths from GTM to other RTO can have different delay values, depending on the architecture used. Using a CAN bus for communication reduces these differences to a minimum. Sharing the same transport media with data (e.g. ethernet) makes difficult to estimate the SM's transit time from the GTM to slave RTO.

The protocol presented for sending the SM could be modified to set the system frequency to a minimum such that $\mathrm{MAD}$ condition is respected. One disadvantage of this approach is that the system can not adapt well to DFN traffic sudden increases (the threshold value being small, synchronization could be lost for some time)

\subsection{Stream Management}

The central concept in a DFF is to model data as streams of information. The main characteristics of a stream are: frequency and sample size. For optimizing data processing, we found necessary to add other features to a stream:

1. Cacheable

2. Allowed composition set

3. Maximum freshness of a sample

4. Compression algorithm

5. Encryption algorithm

\subsubsection{Stream Metadata Structure}

The metadata associated with a stream represents a collection of the respective stream's parameters. This structure contains the following stream parameters:

1. frequency - the time between two consecutive data samples; for variable frequency streams, we just indicate 0

2. size - the size of a sample

3. cacheable - if the stream can be cached and later used

4. composition set-a set of streams to which the stream composes

5. compression algorithm - what type of compression is applied to the stream

6. encryption algorithm - what type of encryption is applied to the stream

7. freshness - for how long a reading is valid; not necessarily linked with the frequency, as some readings could be used for logging

Fully characterizing a stream, the metadata is essential to the data fusion process, by providing all the vital information for stream management. 


\subsubsection{Stream Creation, Halting and Blocking}

This group of functions allows the creation of a stream object starting from a sensor, the halting of that stream and the blocking. All the functions operate on the sensor dependent parameters which encoded in the metadata structure attached to the respective stream object and on the stream source's device id. As stream objects are used to handle stream data, we can regard these functions as basic means for allocating, deleting such objects. The option of blocking a stream is for

\subsection{Data Acquisition}

We can break data acquisition in the following parts: (1) message transmission and (2) data checking. For (1) there are 2 modalities for sending / receiving messages: synchronous (blocking) and asynchronous (non-blocking). An RTO needs to support both modes for receiving messages. We defined the following message receiving possibilities: (1) timeless blocking, (2) timed blocking and (3) non-blocking. For each of these receiving modes we defined functions that take as parameter the buffer that will contain the data and the device id that is supposed to send that data.

Data checking was defined according to stream specification. Therefore, the function DEF_METADATACHECK receives as single parameter the stream's metadata and indicates whether it is valid or not.

Another important part of data acquisition is represented by buffer management. For managing buffers on receiver side we created definitions for: creating a buffer pool, deleting a buffer pool, allocating (creating) a buffer, deleting a buffer, counting the packets in a buffer, getting a packet from a buffer.

\subsection{Data Processing}

Data processing encompasses all the transformations applied on data on a processing node. It is strongly dependent on the RTOS running on the peer for issues such as scheduling and execution time of various procedures.

\subsubsection{Scheduling}

The scheduling addresses two important issues: starting and execution time for a specified method. There are two types of methods: one that execute asynchronously (activated by clients requests) and one that execute synchronously periodically (scheduled with a well specified period).

The scheduling parameters of methods to be scheduled are:

1. scheduling period - represents the time interval between two consecutive executions

2. guaranteed completion time - the amount of time the method is going to complete

3. start time - the time for the first invocation of the method

4. stop time - the stop time of a method

5. error handler - pointer to the error handler for that method

6. error handler guaranteed completion time - the GCT for the method error handler

According to the method's type, we defined functions for scheduling / un-scheduling methods and for halting the executing of methods currently active.

\subsubsection{Merging Streams}

Merging streams performs the final step of data fusion. Its inputs are represented by two processed streams, brought to a normalized frequency and packet form by the Acquisition Layer. Its output is the resulting, fused stream, potentially with its own frequency and data format. For merging we defined only 3 functions: selecting a stream, combining 2 streams and removing a stream, which obviously operate on stream metadata.

\subsubsection{Error Handling}

Our error handling mechanism allows the user to debug its applications in real-time. The most important condition comes from the error handler functions which need to be extremely simple, for having a low guaranteed completion time.

\subsection{Data Forwarding}

Data communication deals with sending the results of local data processing to other peers. The impacting issue is represented by transmission buffer management. This can be done similarly to the buffer management described for Data Acquisition Level.

\section{Conclusion}

In the present paper we specified a framework for writing data fusion applications in a time oriented manner with high flexibility in defining programmer fusion entities supporting complex interaction patterns. We have defined the notion of fusion object and presented its implementation, RTO. We also provided a brief overview of most important functionalities encompassed by our hierarchical data fusion API. As a next research point is to fully implement the data fusion framework and to test it in real autonomous driving conditions.

\section{REFERENCES}

[1] B. Lutz, "Driving is Dead", Fortune Magazine http:/www.fortune.com/fortune/brainstorm $/ 0,15704,644254,00 . \mathrm{html}$

[2] DARPA Grand Challenge, "LA to Las Vegas" http://www.darpa.mil/grandchallenge/

[3] S.B. Gardner, "A Decentralized Data Fusion Framework for Horizontal Integration of Intelligence", Data International Conference on Intelligence Analysis, 2005

[4] D. N. Jayasimha, S. S. Iyengar, and R. L. Kashyap. "Information integration and synchronization in distributed sensor networks", IEEE Transactions on Systems, Man and Cybernetics, 1991, pp.1032-1043

[5] R. Kumar, M. Wolenetz, B. Agarwalla, J. Suk Shin, P. Hutto, Arnab Paul, and U. Ramachandran, "DFuse a framework for distributed data fusion.", Proceedings of the First International Conference on Embedded Networked Sensor Systems, 2003, pp. 114-125

[6] IRIX Ream Time Operating System, SGI, http:/www.sgi.com/products/spftware/irix

[7] QnX Real Time Operating System, http://www.qnx.com

[8] K. H. Kim, C. Subbaraman, "Principles of Constructing a Timeliness-Guaranteed Kernel and Time-Triggered Message-Triggered Object Support Mechanisms", ISORC 1998, pp. 80-89

[9] Douglas C. Schmidt, Aniruddha Gokhale, Tim Harrison, and Guru Parulkar, "A High-Performance EndsystemArchitecture for Real-time CORBA," IEEE Communications Magazine, vol. 14, no. 2, 1997

[10] I. Sommerville "Software Engineering", Addison Wesley, $7^{\text {th }}$ edition, 2004 